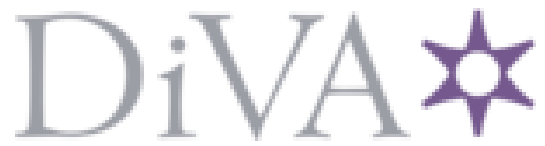

http://www.diva-portal.org

\title{
Postprint
}

This is the accepted version of a paper presented at 2021 13th International Conference on Quality of Multimedia Experience (QoMEX),Virtual, Online, 13 June 2021 - 17 June 2021,.

Citation for the original published paper:

Kelkkanen, V., Fiedler, M., Lindero, D. (2021)

Remapping of hidden area mesh pixels for codec speed-up in remote VR

In: 2021 13th International Conference on Quality of Multimedia Experience, QoMEX 2021, 9465408 (pp. 207-212). Institute of Electrical and Electronics Engineers (IEEE) https://doi.org/10.1109/QoMEX51781.2021.9465408

N.B. When citing this work, cite the original published paper.

Permanent link to this version:

http://urn.kb.se/resolve?urn=urn:nbn:se:bth-21381 


\section{Remapping of Hidden Area Mesh Pixels for Codec Speed-up in Remote VR}

\author{
Viktor Kelkkanen \\ Department of Computer Science \\ Blekinge Institute of Technology \\ Karlskrona, Sweden \\ viktor.kelkkanen@bth.se
}

\author{
Markus Fiedler \\ Department of Technology and Aesthetics \\ Blekinge Institute of Technology \\ Karlshamn, Sweden \\ markus.fiedler@bth.se
}

\author{
David Lindero \\ Ericsson Research \\ Ericsson $A B$ \\ Luleå, Sweden \\ david.lindero@ericsson.com
}

\begin{abstract}
Rendering VR-content generally requires large image resolutions. This is both due to the display being positioned close to the eyes of the user and to the super-sampling typically used in VR. Due to the requirements of low latency and large resolutions in VR, remote rendering can be difficult to support at sufficient $s$ peeds in $t$ his medium.

In this paper, we propose a method that can reduce the required resolution of non-panoramic VR images from a codec perspective. Because VR images are viewed close-up from within a headset with specific $l$ enses, $t$ here a re $r$ egions of $t$ he images that will remain unseen by the user. This unseen area is referred to as the Hidden-Area Mesh (HAM) and makes up 19\% of the screen on the HTC Vive VR headset as one example. By remapping the image in a specific $m$ anner, $w e c$ an $c$ ut o ut the HAM, reduce the resolution by the size of the mesh and thus reduce the amount of data that needs to be processed by encoder and decoder. Results from a prototype remote renderer show that by using the proposed Hidden-Area Mesh Remapping (HAMR), an implementation-dependent speed-up of 10-13\% in encoding, $17-18 \%$ in decoding and $7-11 \%$ in total can be achieved while the negative impact on objective image quality in terms of SSIM and VMAF remains small.
\end{abstract}

\section{INTRODUCTION}

Remote rendering is a technology that can be used to view highly complex 3D scenes on low-powered hardware by rendering on a high-powered server and streaming the content as video to a low-powered client. The client device needs no highpowered GPU, only a strong hardware decoder and network connection, thus there is a potential of reducing cost, power consumption and size [1]. Remote rendering with interactive content is widely available in today's market in the form of cloud gaming. These services already require a relatively low latency, typically $60-120 \mathrm{~ms}$ [2], but remote rendering for VR must take this further. A maximum latency of $20 \mathrm{~ms}$ from Motion To Photon (MTP) is generally regarded as acceptable in VR [2], [3]. There are many latency mitigation techniques available for VR that are used in commodity devices today, for example prediction [4], [5], 2D-image warping [5]-[8] and 3D-image warping [9]-[11]. These merely help to mitigate latency, however, and to quote John Carmack: "It is always better to not have a problem than to mitigate it, so true latency reduction should be aggressively pursued" [8].

Partially funded by the KK Foundation under contract 20170056.

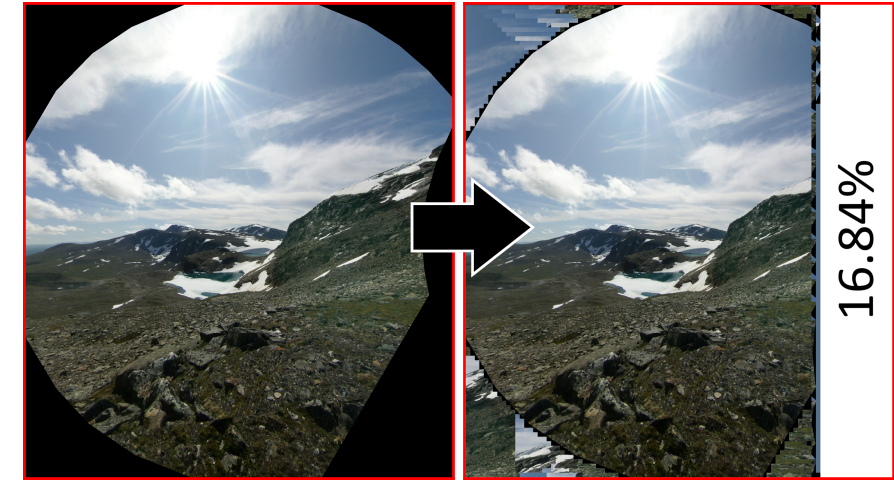

Fig. 1. VR image of the left eye with the unseen HAM in black (left), and a mapping of this image (right) that reduces the amount of pixels by $16.84 \%$.

In this work, a method is proposed which can help reduce the latency of remote rendering for VR. The method is based on the fact that VR images have areas that will remain unseen by the user and are commonly not rendered, see Figure 1 The black area or Hidden-Area Mesh (HAM) consumes 19\% of the total number of pixels on the HTC Vive as one example. This means that the images are $23 \%$ larger than needed, which implies that $23 \%$ extra data must be processed by the encoder and decoder. The concept of a HAM is not unique to the HTC Vive but is applicable to many VR headsets on the market. While we only physically tested the HTC Vive, judging by external screenshots we find for example approximately 19.8\% hidden pixels in the Vive Pro [12], 24.8\% in the Valve Index [13] and $15.1 \%$ in the Oculus DK2 [14]. We propose that by removing the unseen pixels, a speed-up can be gained because less data needs to be moved and processed in the encoding and decoding processes.

The HAM is naturally not a block of pixels that can easily be cut out though, which is why a mapping process must be introduced. This is used to rearrange the rendered image to a rectangle in which black pixels from the HAM are replaced by non-hidden pixels. Thus, the size of the image is optimally reduced by the size of the HAM. The rearrangement of pixels should maintain as much of the original image structure as possible though or may otherwise make the image more difficult to encode which will reduce the image quality. In 
this work, we develop a mapping for the HTC Vive HAM that only causes a small penalty to quality and use it in performance measurements to determine the potential speedup provided by the method. The ultimate goal of the method can be summarized as reducing codec time-consumption while affecting quality as little as possible. In this work, the quality is evaluated by two objective quality estimators (SSIM and VMAF) as they are deemed sufficient for determining the viability of the method. Furthermore, in times of COVID-19, subjective experiments with shared VR headsets would pose the risk of spreading the disease.

The paper is structured as follows: In Section III related work is discussed. Section III presents the design of the experiments and details regarding implementation. Section IV shows how the proposed HAM Remapping (HAMR) performs in a number of tests. The work is summarized in Section $\mathrm{V}$ where also potential future work is discussed.

\section{RELATED WORK}

As previously stated, extensive software innovation in VR remote rendering can be found in the area of latency mitigation, e.g. prediction and warping [4]-[11]. True latency reduction, however, has typically been achieved by hardware innovation, e.g. by hardware-accelerated codecs such as the Nvidia Video Codec [15] and improved wireless LAN protocols such as IEEE 802.11 ad [16] and WirelessHD [17]. However, software innovation may also help in latency reduction, e.g. by utilizing parallel processing in clever ways [18], [19], by reducing the image size with super resolution [20], or by using a reduced pixel density in the peripheral regions [19], [21], [22]. Still, we have not yet seen a method that solely considers the HAM and rearrangement of pixels for latency reduction, which is our gap to fill.

\section{A. Remote Rendering in $V R$}

A number of remote rendering solutions for VR have been presented, both in terms of non-panoramic and $360 \mathrm{VR}$. While HAMR is not applicable to $360 \mathrm{VR}$ (due to their streaming of panoramas that cannot benefit from HAMs), it is intended to improve speed in non-panoramic solutions, e.g. [18] and [23]. In [18], a method for reducing latency in remote VR is presented. In summary, the image is split multiple times and processed in parallel. We also utilize parallel encoding and decoding in this work but with the addition of HAMR.

Note that HAMR is limited to reducing latency originating from the codec. Thus, if the loop-speed is limited by other factors such as Round Trip Time (RTT), e.g. in cloud rendering across the public internet, HAMR is not useful. Its intended use lies in systems with ultra-low latency requirements, where the goal is a latency very close or equal to local rendering, for example systems similar to the Oculus Link [19].

\section{B. Super Resolution (SR)}

SR is a technology that can upscale small images with better accuracy than traditional interpolation methods by using machine learning. Its purpose is similar and could complement

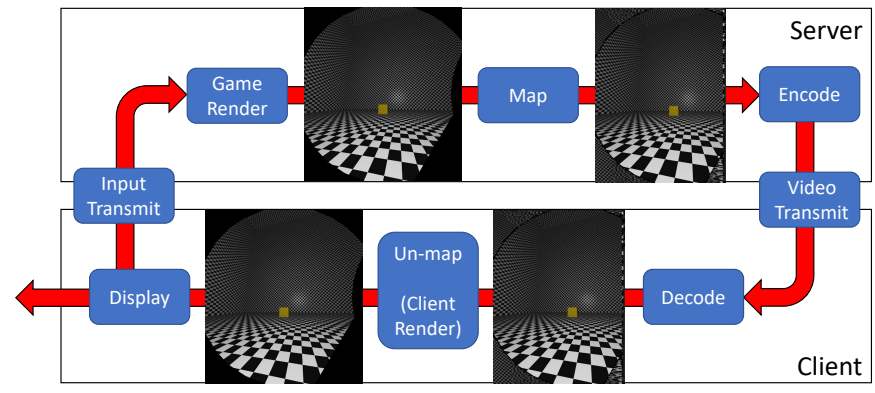

Fig. 2. Overview of the remote rendering loop with included mapping processes and example image of the left eye.

HAMR in future work. The challenges introduced by SR are the processing power required to run the algorithms at sufficient speeds and the need for model training. According to a survey on real-time SR methods [20], the fastest methods at that time (2019), at $\times 2$ up-scaling, required 7.1-21.2 ms per image on an Nvidia Titan X GPU depending on the image data set. At $\times 8$ up-scaling, the fastest method ran at $9.6-29.3 \mathrm{~ms}$. Additionally, [20] shows that the best SSIM of all surveyed methods are in the range $0.9038-0.9797$ at $\times 2$ up-scaling and $0.6369-0.8911$ at $\times 8$ upscaling (depending on the data set).

SR was also used in previous work related to VR using 360-video [24], here, the size of the models were addressed by using micro-models that cover short video segments.

\section{Foveated Encoding (FE)}

FE is a related technology where pixel density or quality is reduced in out-of-focus areas. The approach can consist of reducing the encoder QP-parameter of out-of-focus macroblocks by tracking the gaze of the user [25]. However, while that approach will reduce or optimize the bitrate requirement, it will not significantly impact latency [25]. To reduce latency, the image size should be made smaller by reducing the amount of pixels in non-foveated areas [19], [21], [22].

\section{MethoD}

To test HAMR, a number of mappings are developed and applied in a prototype VR remote renderer. The resulting objective image quality of the mappings are calculated and the speed-up of the best mapping is determined.

\section{A. Codec}

The prototype uses the Nvidia Video Codec SDK 11.0.10 with NVENC as encoder and NVDEC as decoder [15]. Testing was conducted with H.264 and H.265. The client and server programs are written in $\mathrm{C}++$ with the client utilizing OpenGL in rendering and the server supporting both OpenGL and Direct3D 11 (D3D11) to render the game scene.

The encoder implementations are based on the low-latency samples and recommendations from the Nvidia Video Codec SDK, fixed settings are shown in Table I 


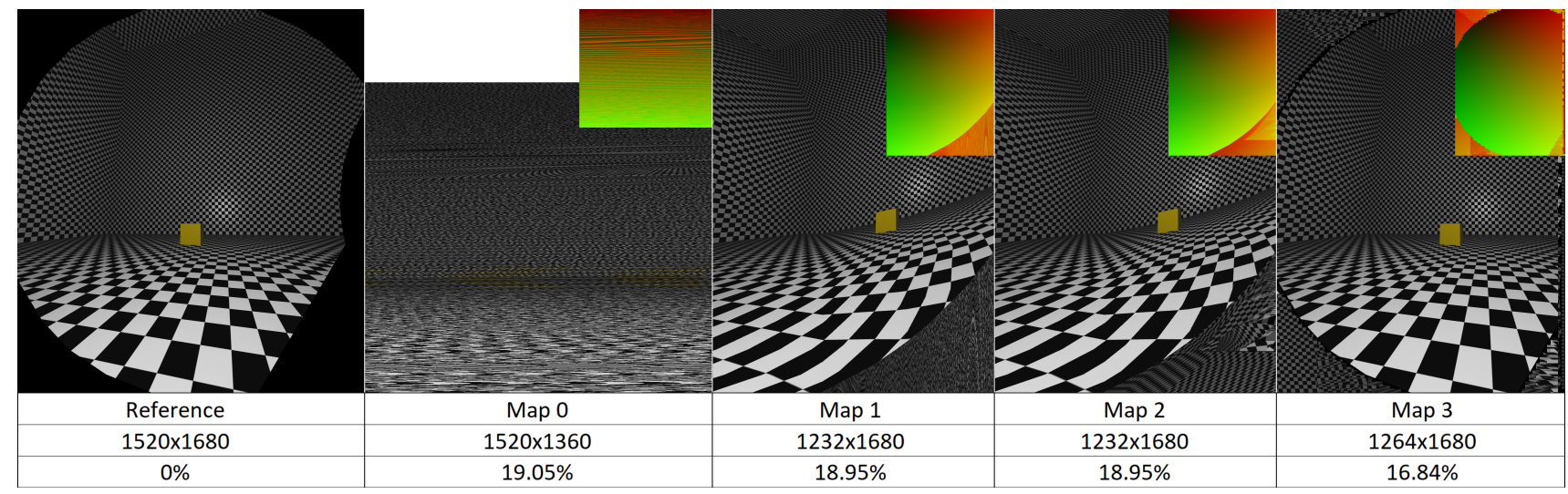

Fig. 3. Reference image and mappings with individual resolutions and total reductions in percentage of pixels. The top right of each mapping shows a thumbnail visual representation of the integer mapping images used in shaders. They contain the $\mathrm{x}$ and $\mathrm{y}$ of each non-hidden pixel in the reference image.

TABLE I

NVENC PARAMETERS

\begin{tabular}{ll} 
frameRate & 90 \\
gopLength & NVENC_INFINITE_GOPLENGTH \\
rateControlMode & NV_ENC_PARAMS_RC_CBR \\
multiPass & NV_ENC_TWO_PASS_QUARTER_RESOLUTION \\
vbvBufferSize & (bitrate $\times 1 /$ frameRate) \\
tuningInfo & NV_ENC_TUNING_INFO_ULTRA_LOW_LATENCY \\
chroma & YUV420 \\
\hline
\end{tabular}

\section{B. Caveats Regarding Pixel Rearrangement in H.264}

1) Discrete Cosine Transform (DCT): DCT of pixel blocks is used in H.264 and other video- and image-compression algorithms to transform the image from the pixel domain to the frequency domain. This makes it unwise to destroy the spatial relationship between pixels since this transformation is done to utilize the internal correlation that exists within local areas of an image. Coefficients in the DCT filters are affected by the spatial correlation of adjacent pixels. Pixels with little distance in between usually have a lot in common, e.g. blue pixels in the sky usually lie close to other blue pixels.

2) Macro Block Sizes: H.264 uses a hierarchical approach to designate macro block sizes ([26], p. 569). $4 \times 4$ pixel sizes are most commonly used but depending on content. Many different combinations can be used up to $16 \times 16$ pixel blocks. Also, since H.264 can use intra-macroblock prediction [27], it makes sense to keep the $16 \times 16$ blocks intact to maintain the compression and quality level after the rearrangement.

3) Motion Vectors: It is also important to keep some larger scale spatial structure intact to not break the motion vector search algorithms. This is also likely the area where most can be gained from making the image smaller. Motion vector calculations are computationally heavy, and even though different methods have been tried, do not scale well [28]. The simple solution is to reduce the number of macro-blocks in a picture, i.e. making it smaller by removing unnecessary pixels. See for example [29], p. 23 for a comparison between a couple of reference encoder implementations. Based on those examples, it becomes obvious that the motion compensation is the major contributor to the total encoding time.

\section{Mapping}

The VR image can be remapped in many ways by utilizing the HAM. Depending on how the encoder functions internally, some of these mappings will be more optimal than others.

Using a mapping will not only result in a quality penalty, but also a performance penalty in the client and server rendering steps. The rendered images must be mapped onto smaller images in a post-processing pass before encoding is conducted on the server. Similarly, the client must un-map the decoded image so it can be sent to the display, see Figure 2 These operations need not be costly however, they can be reduced to essentially one image load and one image store operation per pixel on both server and client. In summary, a mapping coordinate is loaded from a map image, and a pixel from a source image is stored in that coordinate on the destination image. When mapping, the source is the rendered reference image and the destination is the mapped image before encoding. When un-mapping, the source is the mapped image from the decoder and the destination is the image to be sent to the VR driver and display, see Figure 2 The maps are integer images with a red and green channel of 16 bit each, representing the $x$ - and $y$-coordinates of the reference image, see Figure 3 .

The per-eye image size used in this work is based on the default super-sampled resolution of the HTC Vive, $1512 \times 1680$ [30], which is based on its physical display size of $1080 \times 1200$ super-sampled by $1.4 \times$ to account for lens distortion, as is the default when using this headset. However, the exact resolution used in this work is rounded up to the closest multiple of 16 $(1520 \times 1680)$ to avoid cropping in the decoder.

Map 0: The first map (0) is referred to as the naïve mapping because it does not consider the image structure. In this mapping, every non-hidden pixel is moved to the closest hidden pixel starting from the top left. This enables efficient usage of the hidden area but will severely distort the image and thus harm the image quality. Map 0 reduces the amount of pixels by $19.05 \%$. 
Map 1: This map is more refined and based on a number of steps that begin with a warping of the image where every non-hidden pixel is moved to the left if there are hidden pixels to replace. Secondly, every non-hidden pixel is moved upward if there are hidden pixels to replace. This results in a warped image where all black parts are contained to the lower right of the image, remaining pixels are thereafter moved to fill in this black area in the same naive way used in Map 0. The mapping achieves a reduction of $18.95 \%$.

Map 2: Similar to Map 1 but maintains many pixel connections when moving the remainder to the hidden area. Map 2 maintains connections after the warping by moving one column of non-hidden pixels to one row of the hidden area and selecting a new column whenever the space on the hidden row runs out. This keeps connections to some extent but is not perfect due to the differences in dimensions, the last pixels are therefore still squeezed in. Note the difference in the lowerright parts of Map 1 and Map 2 in Figure 3

Map 3: This map is based on the principle of keeping together $16 \times 16$ blocks of pixels as motivated in Section III-B. in order to maintain quality. Furthermore, by not warping the image, most of it will remain identical to the reference image, but there will then be three black areas to fill in, which may reduce the filling efficiency. Due to the use of $16 \times 16$ pixel blocks, some space will be wasted in the round areas around the mesh. Map 3 therefore requires two extra columns of 16 pixels each to fit the remaining blocks, note the 32 extra pixels to the right side of Map 3 in Figure 3 . Map 3 yields a reduction of $16.84 \%$ and is thus not as efficient as the other mappings in terms of space, but is on the other hand expected to provide a significant improvement with regards to image quality.

\section{Experimentation}

To measure the penalty in image quality from using the mappings, tests are conducted where frames are captured as lossless PNG on client and server and made into individual videos of the lossless video format FFV1. The objective image quality is thereafter calculated by comparing the two videos using FFmpeg [31] with SSIM and VMAF (vmaf_v0.6.1).

Finally, the speed of the rendering-loop is measured while running with and without mapping in order to determine the potential latency reduction of using the proposed method.

All experimentation in this work was done with a GTX 1080 as encoder device and an RTX 2070 as decoder, both using driver version 457.51. Note that this is an experimental setup and future client devices will obviously not use VRcapable desktop GPUs but cheaper cards with similar hardware decoding capability, e.g. phones or battery-driven stand-alone HMDs. Network transmission was done over wired Ethernet to minimize network impact in this testing environment.

Two different stimuli were used, one is a basic 3D scene containing colored cube meshes within a room with a checkerboard texture. This is referred to as the Cubes scene and can be seen in Figure 3 The other stimuli uses a cube map from a real-world mountain [32] and is referred to as the World scene, a sample of this scene can be seen in Figure 1

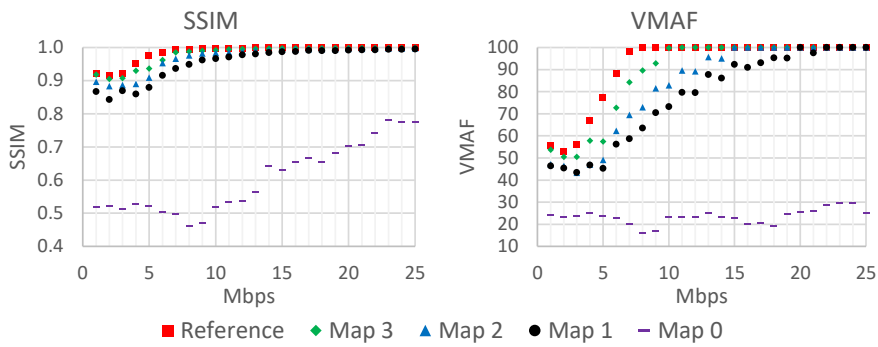

Fig. 4. Objective quality of all mappings and reference case using H.264 in the Cubes scene. One sample per bitrate, constant rotation of $360 \%$ s.

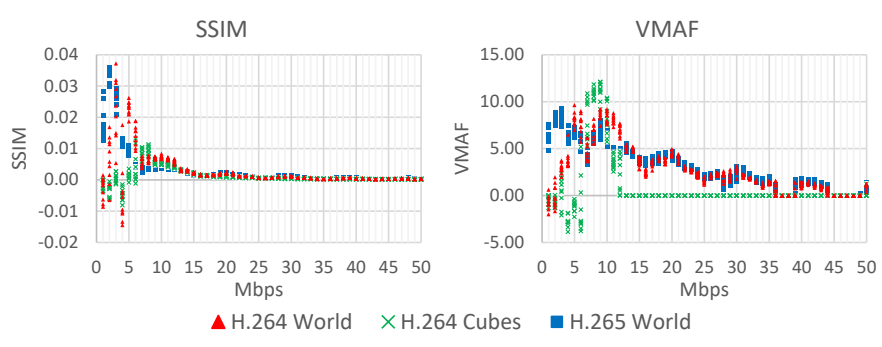

Fig. 5. Differences in objective image quality of Map 3 and a reference. Ten samples per bitrate, constant rotation of $360 \%$ s.

\section{RESULTS}

\section{A. Initial Quality Test}

To compare the general image quality of the different mappings, experiments are conducted where the camera is rotated artificially around the vertical Y-axis by $360^{\circ} / \mathrm{s}$ using all mappings and a reference case without mapping. This rotation emulates a common human head-motion speed which has been found to average at $348 \pm 92 \%$ s [33]. A fast headrotation will make the rendered content move fast, which will make it more difficult to encode, thus increasing bitrate requirements. To measure the effect of bitrate, it is changed during testing from $50 \mathrm{Mbps}$ per eye down to $1 \mathrm{Mbps}$. One frame per bitrate is recorded for quality estimation in these tests, which is sufficient to tell the quality-results apart, see Figure 4. Bitrates between 26 and $50 \mathrm{Mbps}$ did not affect quality noticeably and were cut from the graphs to save space.

Figure 4 shows that the quality produced by the mappings increases with each iteration. There is an exceptional leap between Map 0 and Map 1 and a gradual increase from 1 to 3 . The results determine that maps 1, 2 and 3 converge with the reference on high bitrates and that Map 3 provides the best quality. The VMAF of Map 3 converges at $10 \mathrm{Mbps}$, while Map 2 does so at $15 \mathrm{Mbps}$ and Map 1 at $22 \mathrm{Mbps}$. We note that bitrates under $10 \mathrm{Mbps}$ may not be sufficient for the scenes shown, while the corresponding SSIM and VMAF values clearly indicate differences between the mappings. Obviously, the more a mapping takes video encoding techniques into account, the smaller the differences to the original become, as indicated by SSIM and VMAF values. 
Time consumption, Reference vs Mapping 3

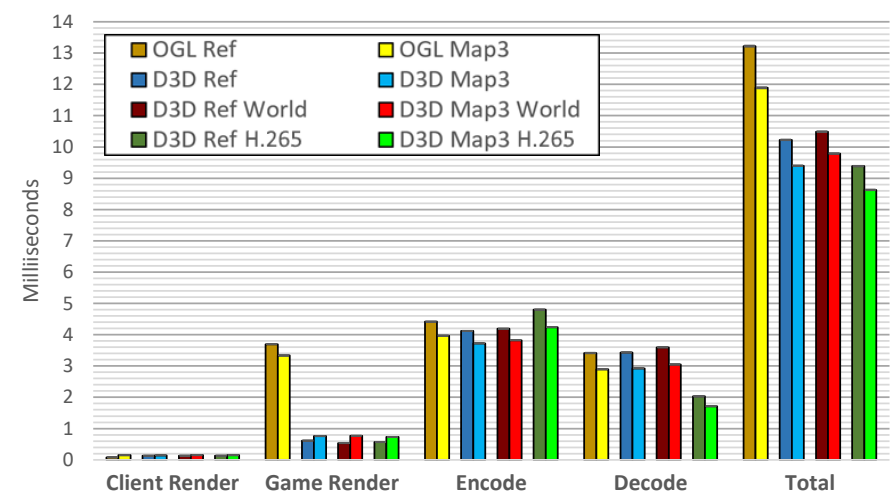

Fig. 6. Time-consumption of Map 3 and a reference in different server implementations and settings. Tests with the World scene used $20 \mathrm{Mbps}$ per eye, all others used the Cubes scene and $10 \mathrm{Mbps}$. Constant rotation of $360^{\circ} / \mathrm{s}$.

\section{B. Quality Testing with Map 3}

The results conclude that Map 3 provides the best quality and is therefore used in further testing. The previously described test is repeated with Map 3 in the Cubes scene and additionally the World scene using H.265. Results from these tests are revealed in Figure 5. The difference in quality when compared to a reference is still small and generally peaks before $10 \mathrm{Mbps}$. After the peak, as bitrate increases, the SSIM and VMAF measurements converge slowly, with the exception of VMAF in the Cubes scene which will reach and stay at zero difference from $13 \mathrm{Mbps}$ and onwards.

While there is a small difference in quality (at adequate bitrates) and thus room for small improvements, Map 3 performs with similar quality to that of the reference-case when provided with sufficient bitrate. According to an interview with Netflix, the Just Noticeable Difference (JND) of VMAF is 6 [34]. Map 3 was able to produce results in that region and below, therefore it is selected for further testing with regard to time-consumption. Note that H.264 and H.265 provide similar results in terms of quality which suggests that both codecs are appropriate for HAMR.

\section{Speed}

In this section, we investigate the potential of Map 3 in terms of speed-up of the remote rendering loop. To measure the speed-up, the Cubes testing scene is used with the same $360 \%$ rotation and $10 \mathrm{Mbps}$ bitrate to render $10 \mathrm{k}$ frames with Map 3 and a reference, respectively. Testing is done with H.264 using the OpenGL and D3D11 server implementations, and H.265 using D3D11 only. To determine whether speed-up is impacted by stimuli or bitrate, the World scene is used in a similar test using the double bitrate of $20 \mathrm{Mbps}$ with H.264.

The average time-consumption of the relevant components with (hardly visible) $99.9 \%$ confidence intervals are presented in Figure 6 and additional details are shown in Table II Evidently, the mapping operations are fast, client rendering was maximally impacted by a $0.07 \mathrm{~ms}$ penalty and server rendering by $0.24 \mathrm{~ms}$. The codec speed-up was large enough
TABLE II

DIFFERENCE IN TIME CONSUMPTION OF REFERENCE AND MAP 3

\begin{tabular}{ll|lllll} 
Server & Config & $\begin{array}{l}\text { Client } \\
\text { Render }\end{array}$ & $\begin{array}{l}\text { Server } \\
\text { Render }\end{array}$ & Encode & Decode & Total \\
\hline OpenGL & Cubes & $+0.07 \mathrm{~ms}$ & $-0.36 \mathrm{~ms}$ & $-0.45 \mathrm{~ms}$ & $-0.52 \mathrm{~ms}$ & $-1.33 \mathrm{~ms}$ \\
H.264 & $10 \mathrm{Mbps}$ & $(+45 \%)$ & $(-11 \%)$ & $(-11 \%)$ & $(-18 \%)$ & $(-11 \%)$ \\
\hline D3D11 & Cubes & $+0.01 \mathrm{~ms}$ & $+0.14 \mathrm{~ms}$ & $-0.40 \mathrm{~ms}$ & $-0.51 \mathrm{~ms}$ & $-0.83 \mathrm{~ms}$ \\
H.264 & $10 \mathrm{Mbps}$ & $(+9 \%)$ & $(+19 \%)$ & $(-11 \%)$ & $(-17 \%)$ & $(-9 \%)$ \\
\hline D3D11 & World & $+0.02 \mathrm{~m}$ & $+0.24 \mathrm{~ms}$ & $-0.37 \mathrm{~ms}$ & $-0.55 \mathrm{~ms}$ & $-0.70 \mathrm{~ms}$ \\
H.264 & $20 \mathrm{Mbps}$ & $(+11 \%)$ & $(+31 \%)$ & $(-10 \%)$ & $(-18 \%)$ & $(-7 \%)$ \\
\hline D3D11 & Cubes & $+0.01 \mathrm{~ms}$ & $+0.16 \mathrm{~ms}$ & $-0.56 \mathrm{~ms}$ & $-0.31 \mathrm{~ms}$ & $-0.76 \mathrm{~ms}$ \\
H.265 & $10 \mathrm{Mbps}$ & $(+10 \%)$ & $(+22 \%)$ & $(-13 \%)$ & $(-18 \%)$ & $(-9 \%)$
\end{tabular}

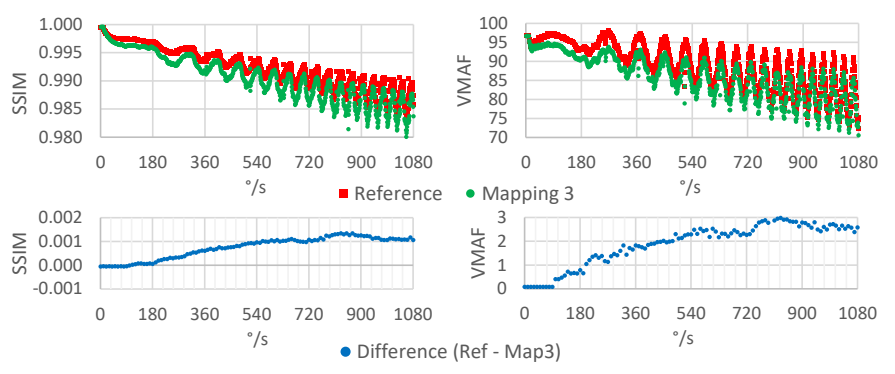

Fig. 7. Absolute (top) and difference (bottom) in quality using the World scene with $20 \mathrm{Mbps}$ per eye while applying a rotation-speed that increases every 10 frames. The wave-like forms in absolute quality are due to the change in view-direction and varying content of the mountain scene.

to overcome this penalty in all tests since the total timeconsumption was reduced in every case. Encoding improved in all tests, between 10-13\%. Decoding improved 17-18\% overall but was significantly faster with H.265. On the contrary, encoding with H.265 was slower, but the total time was still lower for H.265, as seen from the blue and green bars in Figure 6 In total, the introduction of Map 3 yielded a speed-up between $7-11 \%$ depending on implementation and settings.

Note that the reason for the abnormal time-consumption of Game Render in OpenGL is due to NVENC not supporting OpenGL textures as input in Windows 10. An extra memory copy is therefore done from OpenGL to CUDA memory in that implementation, which furthermore explains the decrease that occurs when using a smaller image size due to HAMR.

\section{Different Rotations}

All testing so far has been conducted with a constant $360^{\circ}$ /s rotation, which is approximately the average head-rotation speed of humans [33]. While this speed can be regarded as normal, [35] reports peaks of around $800 \%$, and in-house testing revealed values of up to $1000 \%$ and beyond. While such high speeds may be unpractical and even unpleasant, we here make sure that the mapping will not cause problems at higher rotation-speeds. A final test using H.264 is conducted where the rotation-speed is gradually increased from $0 \% \mathrm{~s}$ to $1080 \%$ with $10 \%$ increments every 10 frames. A constant bitrate of $20 \mathrm{Mbps}$ per eye is used with the World scene. The results of this experiment are presented in Figure 7. The data confirms that the mapping will not fail due to high rotation speeds, at least not at this bitrate. 


\section{Conclusions}

HAMR can indeed be used to reduce latency in remote rendering for VR. Experiments show that an implementationdependent speed-up can be achieved in encoding and decoding. A total speed-up of the entire remote rendering loop of $7-11 \%$ was observed in the presented experiments. The speedup was achieved with little penalty to objective image quality when compared to a non-mapping reference. The quality depends on the specific mapping and could be improved further in future work. Future work may also include testing with humans to determine the subjective quality of the method.

\section{ACKNOWLEDGMENT}

We thank our colleagues in the ViaTecH team for the discussions and feedback and the KK foundation for the funding of the project (d-nr 20170056).

\section{REFERENCES}

[1] S. Shi and C.-H. Hsu, "A survey of interactive remote rendering systems," ACM Comput. Surv., vol. 47, no. 4, May 2015. [Online]. Available: https://dl.acm.org/doi/10.1145/2719921

[2] M. Abdallah, C. Griwodz, K.-T. Chen, G. Simon, P.-C. Wang, and C.-H. Hsu, "Delay-sensitive video computing in the cloud: A survey," ACM Trans. Multimedia Comput. Commun. Appl., vol. 14 , no. 3 s, pp. 54:1-54:29, Jun. 2018. [Online]. Available: https://dl.acm.org/doi/10.1145/3212804

[3] M. Abrash. (2014, January) What VR could, should, and almost certainly will be within two years. [Online]. Available: https: //media.steampowered.com/apps/steamdevdays/slides/vrshouldbe.pdf

[4] U. H. List, "Nonlinear prediction of head movements for helmetmounted displays," AIR FORCE HUMAN RESOURCES LAB BROOKS AFB TX, San Fransisco, USA, Technical paper, 12 1983, https://apps.dtic.mil/sti/pdfs/ADA136590.pdf.

[5] R. M. Taylor II, Virtual Reality System Concepts Illustrated Using OSVR. New York, USA: A K Peters/CRC Press, 2019. [Online]. Available: https://www.taylorfrancis.com/books/e/ 9781315157764/chapters/10.1201/b21598-32

[6] R. H. Y. So and M. J. Griffin, "Compensating lags in head-coupled displays using head position prediction and image deflection," Journal of Aircraft, vol. 29, no. 6, pp. 1064-1068, 1992. [Online]. Available: https://doi.org/10.2514/3.46285

[7] J. M. P. van Waveren, "The asynchronous time warp for virtual reality on consumer hardware," in Proc. 22nd ACM Conf. on Virtual Reality Software and Technology, ser. VRST '16. New York, NY, USA: Association for Computing Machinery, 2016, p. 37-46. [Online]. Available: https://doi.org/10.1145/2993369.2993375

[8] J. Carmack, "Latency mitigation strategies," 2 2013. [Online]. Available: https://danluu.com/latency-mitigation/

[9] W. R. Mark, L. McMillan, and G. Bishop, "Post-rendering 3d warping," in Proceedings of the 1997 Symposium on Interactive $3 D$ Graphics, ser. I3D '97. New York, NY, USA: Association for Computing Machinery, 1997, p. 7-ff. [Online]. Available: https://dl.acm.org/doi/10.1145/253284.253292

[10] F. A. Smit, R. van Liere, and B. Fröhlich, "The design and implementation of a VR-architecture for smooth motion," in Proc. 2007 ACM Symposium on Virtual Reality Software and Technology, ser. VRST '07. New York, NY, USA: Association for Computing Machinery, 2007, p. 153-156. [Online]. Available: https://doi.org/10. $1145 / 1315184.1315212$

[11] E. M. Peek, B. C. Wünsche, and C. Lutteroth, "Image warping for enhancing consumer applications of head-mounted displays," in Proc. Fifteenth Australasian User Interface Conf., ser. AUIC '14. AUS: Australian Computer Society, Inc., 2014, p. 47-55.

[12] C. Elliott. (2020) Vr_ham.jpg. [Online]. Available: https://www.studio397.com/wp-content/uploads/2020/02/VR_HAM.jpg

[13] D. Heaney. (2019) No-mans-sky-ham.png. [Online]. Available: https://mk0uploadvrcom4bcwhj.kinstacdn.com/wp-content/ uploads/2019/08/No-Mans-Sky-HAM.png

[14] Mi. Scnenck. (2013) vrjampi_1.png. [Online]. Available: http:// pixelatedramblings.com/wp-content/uploads/2015/05/vrjamp1_1.png
[15] Nvidia. (2013, August) Nvidia video codec sdk. [Online]. Available: https://developer.nvidia.com/nvidia-video-codec-sdk

[16] C. J. Hansen, "WiGiG: Multi-gigabit wireless communications in the $60 \mathrm{GHz}$ band," IEEE Wireless Communications, vol. 18, no. 6, pp. 6-7, 2011.

[17] WirelessHD. (2010) WirelessHD specification version 1.1 overview. [Online]. Available: https://web.archive.org/web/20190306212958/http: //www.wirelesshd.org/pdfs/WirelessHD-Specification-Overviewv1.1May2010.pdf

[18] L. Liu, R. Zhong, W. Zhang, Y. Liu, J. Zhang, L. Zhang, and M. Gruteser, "Cutting the cord: Designing a high-quality untethered VR system with low latency remote rendering," in Proc. 16th Annual Int. Conf. on Mobile Systems, Applications, and Services, ser. MobiSys '18. New York, NY, USA: ACM, 2018, pp. 68-80. [Online]. Available: http://doi.acm.org/10.1145/3210240.3210313

[19] Oculus, "How does oculus link work? the architecture, pipeline and AADT explained," 11 2019. [Online]. Available: https://developer.oculus.com/blog/how-does-oculuslink-work-the-architecture-pipeline-and-aadt-explained/

[20] X. Li, Y. Wu, W. Zhang, R. Wang, and F. Hou, "Deep learning methods in real-time image super-resolution: a survey," Journal of Real-Time Image Processing, vol. 17, no. 6, pp. 1861-8219, 1992. [Online]. Available: https://doi.org/10.1007/s11554-019-00925-3

[21] S. Lee and A. C. Bovik, "Fast algorithms for foveated video processing," IEEE Transactions on Circuits and Systems for Video Technology, vol. 13, no. 2, pp. 149-162, 2003.

[22] A. Basu, A. Sullivan, and K. Wiebe, "Variable resolution teleconferencing," in Proc. IEEE Systems Man and Cybernetics Conf. (SMC), vol. 4, 1993, pp. 170-175 vol.4.

[23] R. Zhong, M. Wang, Z. Chen, L. Liu, Y. Liu, J. Zhang, L. Zhang, and T. Moscibroda, "On building a programmable wireless highquality virtual reality system using commodity hardware," in Proc. 8th Asia-Pacific Workshop on Systems, ser. APSys '17. New York, NY, USA: ACM, 2017, pp. 7:1-7:7. [Online]. Available: http://doi.acm.org/10.1145/3124680.3124723

[24] M. Dasari, A. Bhattacharya, S. Vargas, P. Sahu, A. Balasubramanian, and S. R. Das, "Streaming 360-degree videos using super-resolution," in IEEE INFOCOM 2020 - IEEE Conference on Computer Communications, 2020, pp. 1977-1986.

[25] F. Frieß, M. Braun, V. Bruder, S. Frey, G. Reina, and T. Ertl, "Foveated encoding for large high-resolution displays," IEEE Transactions on Visualization and Computer Graphics, vol. 27, no. 2, pp. 1850-1859, 2021.

[26] T. Wiegand, G. Sullivan, G. Bjontegaard, and A. Luthra, "Overview of the H.264/AVC video coding standard," IEEE Transactions on Circuits and Systems for Video Technology, vol. 13, no. 7, pp. 560-576, Jul. 2003. [Online]. Available: http://ieeexplore.ieee.org/document/1218189/

[27] VCodex. H.264/AVC intra prediction. [Online]. Available: https: //www.vcodex.com/h264avc-intra-precition/

[28] M. E. Krishnan, E. Gangadharan, and N. P. Kumar, "H.264 motion estimation and applications," Video Compression, p. 28, 2012.

[29] P. Hermansson, "Optimizing an H.264 video encoder for realtime HD-video encoding," 2011. [Online]. Available: http://kth.divaportal.org/smash/get/diva2:432684/FULLTEXT01.pdf

[30] A. Vlachos. (2015, March) Advanced VR rendering. [Online]. Available: https://media.steampowered.com/apps/steamdevdays/slides/ vrshouldbe.pdf

[31] FFmpeg. (2016, April) Ffmpeg - a complete, cross-platform solution to record, convert and stream audio and video. [Online]. Available: https://ffmpeg.org

[32] E. Persson. Måskonåive cube map. [Online]. Available: http://www. humus.name/index.php?page=Cubemap\&item=Maskonaive

[33] U. Röijezon, M. Djupsjöbacka, M. Björklund, C. Häger-Ross, H. Grip, and D. G. Liebermann, "Kinematics of fast cervical rotations in persons with chronic neck pain: a cross-sectional and reliability study," BMC Musculoskeletal Disorders, vol. 11, no. 222, Sep. 2010. [Online]. Available: https://doi.org/10.1186/1471-2474-11-222

[34] J. Ozer. $(2017,9)$ Finding the just noticeable difference with netflix vmaf. [Online]. Available: https://streaminglearningcenter.com/codecs/ finding-the-just-noticeable-difference-with-netflix-vmaf.html

[35] P. Fuchs, Virtual Reality Headsets - A Theoretical and Pragmatic Approach. University of Oulu, Finland: Cambridge University Press, March, 2019. [Online]. Available: https://books.google.se/books?id= IfPJDwAAQBAJ 